\title{
Lineamenti di una Moderna Lotta Antifrana
}

\author{
A. Bellutgi (*) \\ Ricevuto il 18 Maggio 1965
}

\begin{abstract}
Riassunto. - L'A. inizia col rilevare l'alto contributo di calamita' lavoro, spese che le situazioni di frana (numerosissime e ravvivantesi), com. portano in Italia.

Accenna subito dopo che, purtroppo, non si tiene nel dovuto conto le pur innumeri "zone sospette" che si maturano quasi sempre con segni premonitori, lasciati a se stessi, senza nessuna "Istituzione specifica permanente", dedita al rilevamento (triangolazioni aggiomate, clinografie, tensio e pressiografie, spie estensinetriche, vibrografie, ecc.).

Si illustrano i concetti di "autogenesi di frana ", autoctona o allactona, "ipocentri di frana" che si attivano post-incubazioni di antotensioni (equi. libri di minima stabilità), da cui dipartono quelle "direttrici di rottura" che, nei corpi elastici uniformi, si identificano con le "tensioni principali elastiche " v. Mastrodicasa: "Dissesti statici delle strutture edilizie " Hoepli, 1965 .

Definito geomeccanicamente il "suolo reale", si mostra come sono traslatabili i risultati ottenuti - con presupposto bidimensionale dei \& sistemi piani di tensione" - ai solidi tridimensionali rocciosi. Il presup posto bidimensionale non è affatto limitativo, e la relativa teorica deduttiva si utilizza in pieno, tenendo conto degli "ipocentri di frana", una nuova prassi perciò per risalire alla "superficie di scorrimento " dei massi franosi (non più induttivamente come si fa quasi sempre oggigiorno).

L'A. espone i criteri (numerosi e cruciali), per ben stabilire i "breals'" di frana, misure e dati permanenti, occasionali, formulare le condizioni di stabiliti, in base a leggi geomatematiche, intensificare la ricerca ipocentrale, fino a pervenire possibilmente ad un "presagio di valanga" (con vibrografi, analisi fotoelastiche, ecc.) che dia tempo di applicare soprattutto i metodi moderni di ristrutturizzazione elettrogeocinetici.

Vengono a grandi linee illustrati tali metodi che vanno, più propriamente, dall'elettrogeosmosi agli elettrogetti (EGE - iniezioni elettriche di leganti indurenti in situ, postiniettati), ai sistemi piezoelettroiniettivi (elettrosilicatazioni), che amplificano i "domini trattabili dei suoli ", "ranges" reologici, litologici, porosimetrici ecc.
\end{abstract}

\section{(*) Prof. Ordinario di Fisica Terreslre}


Dopo un rapido esame critico delle vecchie "iniezioni di cemento" per macrocolmate di cavità, fessure, diaclasi, si mostra come queste possono essere utilizzate con l'impiego dell'elettrogetto integrale ", brevettato e applicato dall A., negli CSA e in altre parti del mondo e impiegato sotto altre forme sopratutto nei Paesi Orientali, particolarmente in Russia.

Il "Consultants Bureau Enterprises, Inc. di New York, da tempo si è deciso a tradurre dal russo Opere che riguardano tali argomenti, quasi del tutto sconosciuti in Italia, e naturalmente non impiegati su vasta scala.

1. Le situazioni di frana, rottura di suoli, rilievi, distacchi di fondo, di ciglio, di pendio, crolli regionali, locali episodici o no, soliflussi, viscorrimenti (tante forme dislocativo-distruttive di masse terrose), trovano in Italia un alto primato di calamità.

Sorvoliamo sulle retoriche tecniche o descrizioni di tante sistematiche di "landslides ", quel che occorre è orientarei su tali fenomeni, approfondire la "franogenesi " per interventi tempestivi "geoterapeutici ".

Non è mai apparsa nel nostro Paese una vera e propria letteratura informativa in tal senso (a parte l'opera storica dell'Almagià) e tanto meno si è pensato ad una lotta antifrana, preventiva, continuativa, all'infuori di tardivi, più o meno inutili, interventi di emergenza a carattere transitorio, con trascuranze poi manutentorie: né si dà peso, in tempo utile, alle innumeri manifestazioni telluriche sospette che incombono minacciose nelle nostre contrade e che si vengono maturando quasi sempre con segni premonitori, (sono i slide-blocks, fall-rocks, debris-slide, debris-falls, creeps, fluages, rocks-slide), neoformazioni spaziotemporali per cause varie, sovraccarichi naturali o no, asimmetrici, rilievi positivi o negativi, o sbilanciati, o fermi, o migranti, in periodi formativi di squilibri a lunga o a breve gestazione.

Intanto la "franosità " tende, per legge naturale, ad incrementare i propri eventi di dissesto (in numero $\mathrm{e}$ in entità), per progressive accentuazioni di equilibri indifferenti o di instabilità.

La Fig. $1 a$ è una tipica superficie di scorrimento (s.d.s.), a curvatura uniforme, $A$ è la frana superficiale, $B$ il terreno in posto.

Le s.d.s. $C_{1} C_{2}$ (v. Fig. $1 b$ ) poggiano sulla $C_{3}$ (s.d.s. principale), trascinante un ripiegamento $V$ di testata dello strato base $B$.

Nessuna roccia è immune dalla tendenza al moto totale o parziale verso il basso, vuoi con velocità talmente piccola da considerare l'anno, il lustro, quali unità di tempo (paleofrane), vuoi con velocità istantanee.

Distaccandosi la massa $A$ rimane in posto la $B$ con superficie a forma di conca. 
La frana $A$ va a depositarsi a valle del pendio sollecitando con il proprio peso il preesistente modello.

Configurazione varie di superfici di scorrimento (s.d.s.).

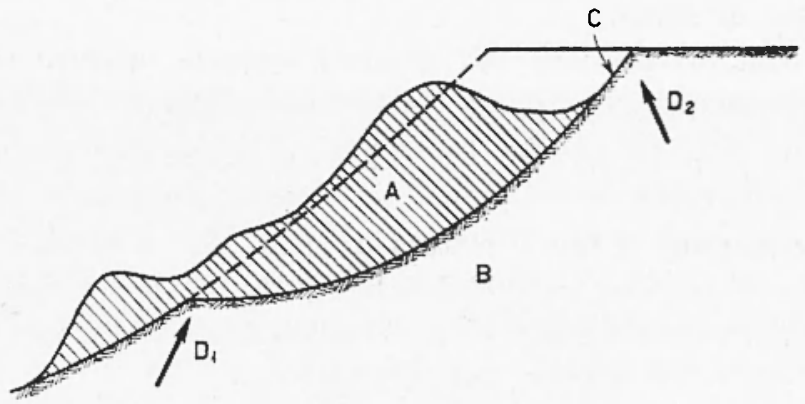

Fig. la - Superficie di scorrimento a curvatura uniforme $A=$ Cono di terreno franato. $-B$ - Terreno in posto. $\gamma=$ Superficie di scorrimento $-D_{1}, D_{2}$, zone di trattamento elettrico più agevoli.

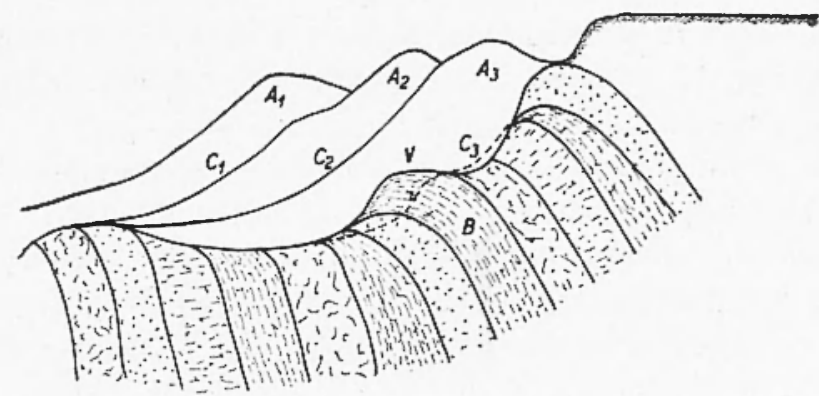

Fig. $1 b$ - Superficie di scorrimento a curvatura disuniforme Elementi di una frana: $A_{1}, A_{2}, A_{3}=$ Zone franate. $-C_{1}$, $C_{2}=$ superficie di scorrimento dopo il franamento. $C_{3}=$ Prima superficie di scorrimento irregolare con ripiegamento parziale di strato $B$.

L'acqua dei pori di questo substrato viene perciò compressa, e poiché essa può defluire nel terreno, più o meno lentamente, ne derivano riduzioni d'attrito nei contatti, avviamento di "frane secondarie", a frane residue ", "frane gemelle " se più discoste, "frane relais ", e queste si possono ripetere più volte, intervallate anche da anni, in coincidenza prevalentemente con le annate umide e piovose.

La "variabile tempo " (questa regina di tutta la geologia, ad azione costante lentissima o rapidissima), fatalmente deve pronunciarsi, là 
dove era stata esclusa dalla prepotente abitudine statica (è la vitalità dinamica delle masse che si ripercuote sia sulle qualità intrinseche dei materiali, che sul modo d'agire dei carichi, che sulle "geoossature", a solidarietà dei legami redenti, a pericolose localizzazioni e conseguenti esasperazioni di sforzi.

Oltre che inventariare tali geostati sospetti, occorre aggiornarli nelle loro evoluzioni, con opere permanenti "d'ascolto tecnico ", dalle triangolazioni alle clinografie, tensiografie e pressografie (spie estensimetriche), vibrografie, esami periodici idrologici, stati climatici e microclimatici, stagionali, ecc.. .

L'uso dei clinografi, largamente diffuso in Giappone, è stato introdotto in Italia, per l'analisi delle situazioni geodinamiche delle dighe, dal Prof. Pietro Caloi, autorevole Presidente dell'A.G.I.

Le "fotoclinografie " ci aiutano a riconoscere moti di varia natura di assestamento tra i più lesivi, come i rotatori di masse, conseguenti in genere a deformazioni elasto-plasto-viscose, per vicende di carichi statici, endo-eso-termogeo-dinamici (impact loads).

Non sammo mai troppe le "diagnosi” da compiere sul "corpo geologico ", malato o apparentemente sano, dapprima orientative, dagli aspetti appunto più pressanti a quelli appena avvertibili di instabilita, siano essi potenziali, che in atto.

E alle prime indagini di rito geo-idrologiche, morfo-topografiche, (vallività scoscese, denudazioni, strati a sostegno-manco, isorientamenti di strati-pendenze, stratificazioni a franapoggio, fillings eluviali, diluviali, forme morbide o ondulate di terreno, sensibili, sconnettibili, alterabili, evoluzioni delle curve ipsografiche, dei profili di equilibrio a mass-wasting-devastazione), si dovranno aggiungere subito dopo le più attente cripto-ricerche di stress-history (paleo e neotettonismi, vergenze e migrazioni di pieghe, subsidenze, ascensionalità o depressività d'orogreni, dia e epigenesi, metamorfismi).

Sono molti i "dati di fatto" che occorrono (di osservazione a di misura) per risalire razionalmente alle vere cause agenti di frana: dalle alterazioni geo-morfologiche (Heim), alle attitudini negative di resistenza al moto (Penk-Pollack): incapacità di contrasto all'azione di forze endo-esogene, crono-topiche: formulare quindi piani di lotta d'emergenza dapprima, di cauto risanamento poi, ciò che gli Anglosissoni raggruppano in due aspetti fondamentali: "removal of material likely to stip ", and "improvement of soil properties".

Tutte le frane "autoctone" o spontanee, "alloctone" o a sollecitazioni esterne, conseguono, se libere di evolversi, il loro epilogo 
A. Belluigi

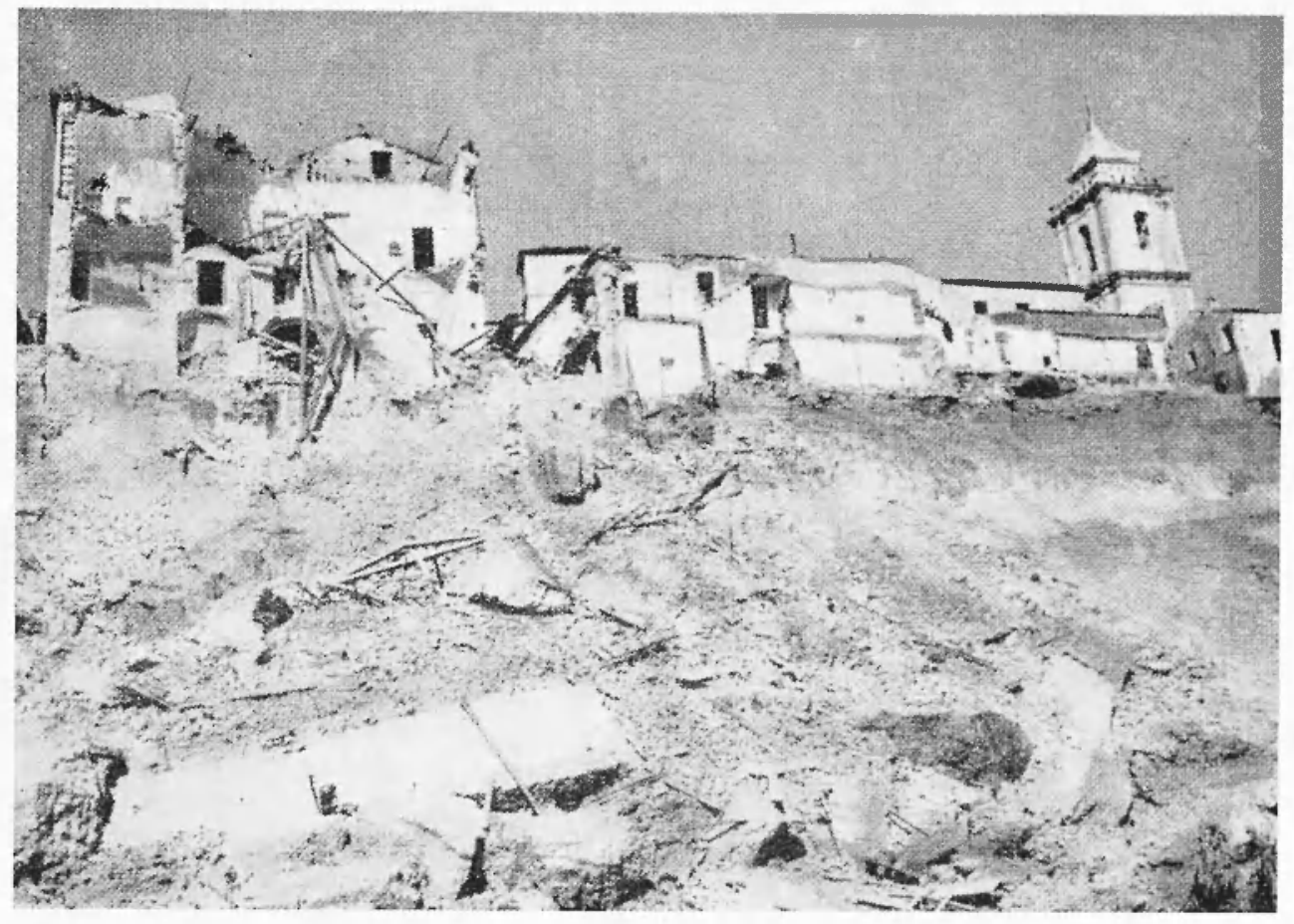

Foto 1 - La frana di Vasto -1956.

A letto di conglomerati e arenarie affioranti giacciono le insidiose argille plioceniche, dal caratteristico colore azzurino. 
di caduta, di collasso e per confluenza di più squilibri (impalcatura) e per vicissitudini sollecitanti, dalle statiche alle dinamiche, dagli inizi deformativi, spostamenti embrionali, ritiri, contrazioni, rigonfiamenti, genetiche tutte spazio-temporali a sé stanti, o reciprocamente più o meno correlazionate.

Carico in equilibrio e in moto

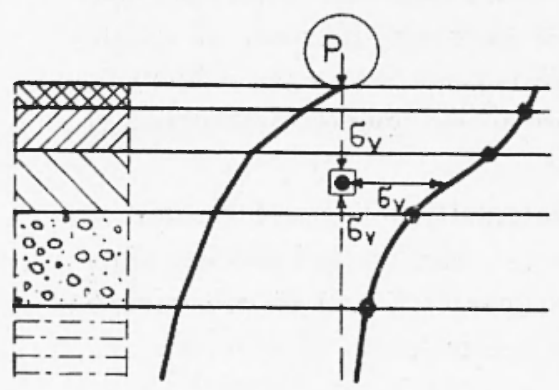

Tensione statica

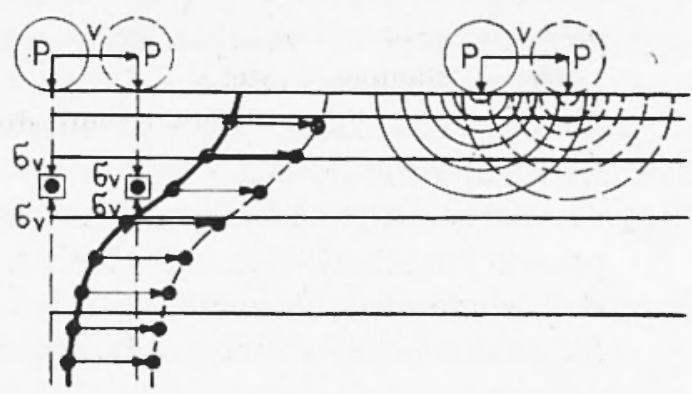

Onda lenta di tensione
Onda rapida di tensione

Fig. 2 - Presagio di valanga. Onde lente, rapide, d'urto, di tensione meccanica, "Ventaglio" di compressione.

Variazione di tensione $m$. per carico $P$ (statico, o in moto), su un terreno sedimentario (a più strati sovrapposti paralleli). "Onda lenta n ed "Onda rapida " di tensione meccanica per carico in moto. Nel "mezzo ipocentrale di frana" si attuano 2 stati cinetici diversi (nelle "fasi condensate "), delimitati dalla superficie ondosa, che in "percussione" appare quale discontinuità di la specie (onda d'urto), mentre nella fase formativa delle solle. citazioni agenti (che non danno luogo allurto), possono provocare " treni d'onda" con discontinuità elastiche seguite da "ventagli " compressivi. Particolare importanza acquista il "numero di Mach ", e la cosidetta "riflessione di Mach $\%$.

All'، autogenesi di frana " pre-distacco e pre-compenetrazione, sovrastano fenomenologicamente incubazioni di "autotensioni " elastoplastiche, equilibri di minima stabilità, deformazioni di varia natura, dalle meno avvertibili dei sedimenti per "vortiefen " fino al dirompente " the break ", " rottura incipiente ", relativi "squeezings " pigri, rapidi, o precipiti, alle "failure lines" traiettorie lesive.

Basta riflettere che un semplice carico statico esercita funzioni compressive, a trazione, di taglio orizzontalmente e verticalmente sul supporto, con sollecitazioni ben delimitabili, tensioni costanti per un 
determinato periodo di tempo, che tendono però ad instaurare deformazioni progressive.

Se poi il carico si sposta, il diagramma delle tensioni si sposterà provocando un'onda lenta o meno di tensione, raggiungente un massimo, per poi diminuire in funzione dell'entità del carico e della durata applicativa (v. Fig. 2).

Tali instabilità incipienti maturatesi, si possono ravvivare per esosituazioni occasionali di carichi variabili, climo-termo-igrometriche, stagionali (tempi di valanga), accelerandosi la neoformazione di quelle " direttrici di rottura " che, nei corpi solidi elastici uniformi, si identificano con le "tensioni principali elastiche", come avviene nei "dissesti statici di strutture edili ".

Nel "settore dei dissesti statici tridimensionali geo-meceanici ", possono formularsi teorie equivalenti a quelle elastiche del sistema sforzi e deformazioni, immutabile con la rotazione degli assi di riferimento (invarianze perciò a carattere isotropico o scalare).

2. La consistenza del "suolo reale " è fondamentalmente geo-meccanica, "stress conditions " rappresentabile in cerchi di Mohr, inviluppi di rottura, "curve intrinseche ".

Da aggiungere l'altro aspetto costitutivo geo-idrologico (bifasicità dei suoli quali "sistemi idraulici »), il tutto riducibile in un'unica consistenza, un'equivalenza geomeccanica da rintracciare o modellare nella sua geotipicità.

Le tensioni che appaiono in una massa terrosa (e i terreni sono sempre sotto tensione) per effetto del peso proprio, sovraccarichi, eventuali spinte idrauliche, impregnazioni fluide, sono suscettibili in una certa misura, di definizioni analoghe a quelle che si dànno nella teoria dell'elasticità.

E possibile definire una "tensione ", dalla forza agente su un'areola infinitesima comunque scelta nella massa in esame, quale rapporto tra essa e l'area stessa, decomponibile in una componente normale all'areola e in una tangenziale.

Lo sforzo di taglio si scompone a sua volta in due parti, l'una dovuta all'attrito interno, l'altra alla coesione.

Non è facile misurare tali parametri, a causa della loro dipendenza, oltre che dalle caratteristiche fisiche e chimiche del suolo, dalle condizioni ambientali, atmosferiche, dalle tensioni stesse cui il terreno è sottoposto. Coesione e attrito interno dipendono sempre dallo sforzo normale, sono suscettibili di variazione nel tempo, per fenomeni vari, e 
i valori che si desumono sono medi e indicativi di quanto accade nella realtà.

Dovendo "prevedere " ciò che genericamente si chiama "frana", ed "evitare " il pericolo che tali moti si producano in un terreno in equilibrio, dobbiamo necessariamente rifarci alle alterazioni dei parametri di resistenza al moto, e sapere per una data condizione di carico, quali tensioni interne si pronunciano, e verificare se esse superano o meno i valori accettabili delle tensioni resistenti.

La "frana " dipende da ciò, è un effetto somma spaziale prolungato nel tempo, che inizia con "rotture locali ": là dove le tensioni debordano, i parametri di resistenza vengono profondamente alterati, in modo che la "rottura" si propaga fino a coinvolgere (lentamente o no) intere masse.

La variazione nel tempo di tali coefficienti impone il loro riconoscimento aggiornato.

A che scopo le classifiche post-evento, i criteri cinematici del Braun (rilievi di velocità di moto franoso), quelli della composizione del mate riale rimosso, delle proprietà fisiche, dei tipi di scivolamento (lento, rapido, esplosivo, globale, parcellare)?

Ciò che conta è accertare le cause di rimozione d'equilibrio, alterazioni, scoesività, lubricità, scuotimenti per ritmie varie, oscillazioni termiche comprese, spinte, variazioni idrostatiche, metamorfizzazioni, idrodinamismi, denudazioni incisive basali, inumidimento di superficie di contatto (spechi di slittamento).

La " concretezza meccanica " del suolo si riassume in una complessa sollecitazione totale agente (3 sforzi o tensioni normali, 6 tensioni di taglio, 4 sforzi scalari-pressione interstiziale d'acqua nei pori, pressione d'aria tellurica, pressione osmotica di sali disciolti in acqua tellurica, entropia del sistema-).

A questo sistema di sollecitazione totale corrispondono deformazioni comprendenti un "tensore incrementale" della deformazione totale a 9 componenti, insieme a 3 deformazioni scalari, 2 per variazioni fluide, 1 per situazione entropica.

In assenza di sollecitazioni dinamiche, i tensori dello sforzo totale e relative deformazioni risultano simmetrici, comprendono ciascuno 6 componenti.

Così in un mezzo omogeneo-isotropo gli assi principali delle tensioni e deformazioni coincidono, e ciascun tensore simmetrico può essere espresso con 3 valori principali di ciascuna di esse, insieme con la posizione degli assi principali, 4 deformazioni scalari, che si conglobano nelle "geoequivalenze". 
Nella prassi moderna di "rilevamento franoso" si tiene conto di tutto questo, è vero, ma sovente in un modo del tutto particolare, insufficiente, causa l'omissione ricostruttiva meccanogenetica dell'inizio di rottura (the break).

Tale omissione "preclude " in genere l'impiego del metodo più razionale nei riguardi della ricostruzione delle stesse traiettorie di propagazione di frana, prescindenti dall'origine del riferimento, e tutte le opere pur fondamentali del Terzaghi, Bendel, Taylor, Fellenius: “Erdstatische Berechungen ", ne risentono, sorrette come sono, da casistiche (tentativi).

La fenomenologia fessurativa per essere compresa non deve prescindere dalla fase incipiente, dalla elementare enucleazione genetica di rottura (un polo di riferimento) che fa corpo con il resto, ed è determinante nel tempo, nelle forme ed evoluzioni.

È il "momento della quantità di moto" rispetto a quel polo (fisso o mobile) che serve per risalire alle equazioni cardinali geo-dinamiche.

Occorrerà qui un sempre maggiore circostanziato corredo di saggi, sperimentazioni, non troppo indispensabili invero, nei processi induttivi.

Preoccupazioni del genere infatti possono attenuarsi nelle ricostruzioni per tentativi, che al massimo sfociano in risultati possibilisti, valevoli (al massimo) quali "ordini di grandezza ".

Per convincersi della funzione determinante della "fase incipiente di rottura " basta riportarsi, oltre il già detto, all'enunciazione stessa del " suolo reale " o " corpo geologico ", una "consistenza iperstatica ", per sovrabbondanza di vincoli interni che ne collegano le varie parti (vincoli efficaci o no): struttura volumetrica pertanto condizionata a varie forme di resistenza che sovrastano la rottura, da quelle più generali (Grashof - Saint Venant) a quelle più particolari.

I suoli ci offrono, ̀̀ vero, tante incognite meccaniche in numero ben maggiore delle condizioni d'equilibrio rintracciabili (per l'invisibile regime interno di tensione), strutture staticamente indeterminate, che pur hanno una loro tendenza naturale all'instabilità, per vetustà, snervamento, ecc...: tutto questo perciò può aiutarci a formulare quesiti atti a ricondurre tali strutture alla determinatezza statica.

Il rilevamento nell'ambito di "rottura iniziale " che racchiude in sé la spiegazione del resto, può non avvenire a causa delle tensioni principali massime o dilatazioni debordanti, potendo il corpo geologico sopportare tensioni ben maggiori (le cosidette "tensioni principali ideali », e questo a seconda delle incidenze di ulteriori sollecitazioni agenti 
di segno diverso, variamente distribuite, più o meno prossime tra di loro): detto rilevamento apporta perciò un contributo decisivo per l'impostazione univoca di quesiti così complessi.

3. La teoria sulla "origine » e "direttrici fessurative », in solidi piani in equilibrio, col presupposto dei sistemi piani di tensione, atta a stabilire con semplici procedure grafo-analitiche le "traiettorie di rottura, è traslatabile ai solidi tridimensionali rocciosi equivalenti.

Basta pensare che se nei suoli reali le tensioni sugli elementi superficiali della stella dei piani accusano direzioni qualsiasi, esistono tuttavia direzioni principali tali (analogamente appunto a quanto si avvera nei sistemi piani) che gli elementi ad esse normali sopportano soltanto tensioni normali (tensioni principali), aventi perciò la proprietà che una di esse è massima e una è minima.

Cosi si deduce (che è poi un fenomeno ricorrente nelle terre) la proprietà, relativa ai solidi piani, che le "linee direttrici fessurative " $e$ " quelle delle tensioni principali ", nei punti di tangenza, si tagliano sotto un angolo diverso da quello retto.

Per stabilire la traslatabilità ai solidi rocciosi tridimensionali equivalenti, ed evitare le difficili impostazioni generali, ci si può avvalere delle analogie fra linee isostatiche o traiettorie delle tensioni principali e le linee di forza elettrica, nonché le ricostruzioni, in luce polarizzata, delle stesse isostatiche, per risalire agli scostamenti anomali di forma dell'isolinee, necessari per i calcoli approssimati, che qui non riportiamo.

Dalla sintomatologia d'instabilità di un "geostato ", sua entità e sviluppo, rinvenimento di qualche causa tra le più appariscenti e attive con eventuale relativo intervento di emergenza, soffocamento dei segni premonitori più inquietanti (sistemato provvisorio), si passa subito dopo alle fasi di accertamento di dettaglio e cruciali.

Dai pesi specifici, volumetrici, limiti di Atterberg, percentuali contenutive di acque, carbonati, solfati, dai dati più comuni di accertamento, si accede a quelli più elaborati: all'analisi granulometriche (forme, dimensioni), microscopiche, setacciamenti, elutriazioni, proprietà dei pori (porosità intergranulari, primarie e secondarie, aperte e chiuse, stabili o istantanee, "porosità cinematiche "), relativi istogrammi: in sostanza a tutte le possibili conoscenze che le tecniche moderne ci permettono di raggiungere.

I rinvenimenti delle aliquote dei "gruppi fini efficaci granulometrici " ad alta reattività, associabili a superassorbimenti o superidrofilie 
nei suoli incoerenti, ci portano a risalire alle possibili carenze di «attrito statico " naturale, localizzare i contatti o i piani in formazione di slittamento, le superfici incipienti di rotolamento o afrizionali.

Sono queste "lubricità geiche " in tutti i tipi di giacitura di roccia (dalle connesse, coerenti - alle sciolte friabili), i fattori maggiormente determinanti i collassi rovinosi.

Diagrafie cicliche delle porosità-pressione, portano ancora ad approfondire le cause efficienti di frana, connesse queste con il depotenziamento dell'" attrito cinematico".

Pressioni in aumento, fino ad una decina di atm. ad esempio, comportano porosità diminuenti; recedendo poi tali pressioni le porosità si ripristinano parzialmente, a meno d'isteresi, " isteresi decompressiva ", indice di sensibilizzazione meccanica della porosità, o capacità variantiva di " attrito cinetico".

Si pensi che un fango argilloso, a volume dei pori $80-90 \%$, a $1000 \mathrm{~m}$ viene compresso con l'11\% dei pori, col $3 \%$ a $2.000 \mathrm{~m}$, e l'elasticità oresce con più rapidità della densità.

Effettuati rilievi, analisi, prove, elaborazioni nella maggior misura possibile, resta la "formulazione geomeccanica " dei solidi geologici equivalenti, non già per tentativi, ma su dati di fatto essenziali conferenti un'alta approssimazione rispetto alla realtà operante.

$\mathrm{Su}$ questi "equivalenti dedotti " si riassorbono e si ridistribuiscono i motivi principali delle sparse consistenze, sfrondandoli dalle molte incidenze secondarie o apparenti.

Tenendo conto di essi, si potranno progettare "risanamenti efficaci ", antagonisti ai dissesti, ed idonee procedure operative atte a conseguirli.

L'eliminazione spinta delle varie casuistiche ci allontana dai falsi scopi, tendendo questi talvolta, addirittura, ad accelerare $\mathrm{i}$ dissesti.

In fondo questa via d'"indagine" non è poi del tutto nuova, in quanto i pionieri s'attennero proprio scrupolosamente ad essa, sia pure in casi più elementari, a cominciare dal principio del "taglio obliquo " (shear failure) di Napier Daubréé-Jeffreys, dai comportamenti elastoplastici del Griggs, alla genesi di cedimenti elasto-plastici (fasi transitorie) per inconsistenza parametrica, o impalcatura snervata, deformata, cause cospiranti sollecitazioni di taglio e normali, quasi sempre legate al teinpo.

Certo a causa della grande varietà di fattori e circostanze confluenti nella genesi e sviluppi di frana, occorrono talvolta incidenze di situazioni inavrertibili (nonostante tutti gli accorgimenti usati), quindi la riesumazione di congrui "equivalenti geo-meccanici", per quanto pre- 


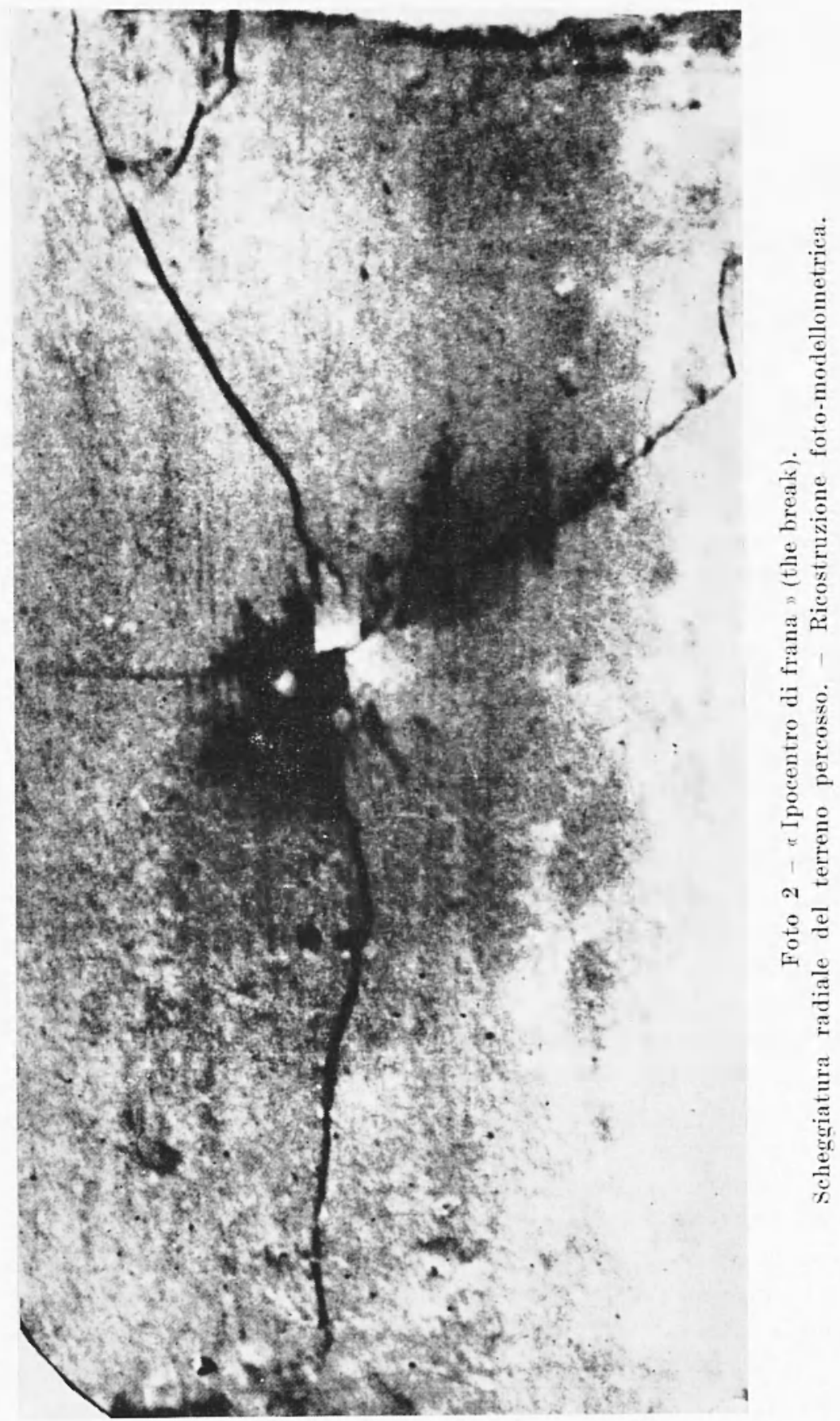


cisa e attenta, può porgere il fianco a manchevolezze. Ma, si noti, queste saranno sempre in numero minore, che non altrimenti, là dove le "tecniche geoinduttive" preseindono addirittura dai dati di struttura; mentre col " pressapoco " o con "l'ordine di grandezza " che si raggiunge, non si potrà mai validamente combattere il flagello in agguato, che poi opera da sé in modo costante, preciso, circostanziato.

Dobbiamo dunque risalire ai Coulomb, ai Rankine, ai tanti altri insigni iniziatori, per ritrovare i primi razionali schematismi deduttivi, le classiche leggi di equilibrio per scarpate, pendii, spalti, per cui, stabilendo certi rapporti quantitativi tra grandezze geometriche e fisicomeccaniche, si stabiliscono sicure condizioni di stabilità, leggi geomatematiche vere e proprie.

E purché tali leggi si rispettino, tutti i "rilevati " potranno permanere ben saldi, senza alcun sostegno, senza interventi artificiali di sorta. Altrimenti si potranno rintracciare, in base alle leggi stabilite, i motivi di manchevolezza, gli stati deficitari (in potenza, indiziali, avanzati), per passare infine al risanamento proprio di quei parametri alterati costitutivi o di quelle impalcature cedenti di cui tali parametri (anche se indenni) fanno parte.

In questo modo sono stati dedotti una quantità di "condizionamenti " di "geostabilità ", prescindendo da concomitanze secondarie o subturbative, con opportune sistemazioni geo-architettoniche o dei parametri alterati contemplati nelle leggi prestabilite.

Sono queste leggi dunque da rintracciare, dai casi più semplici ai meno semplici, subordinare ad esse le progettazioni di idonei interventi, si chiamino esse equazioni di lavoro d'Ivanoff, equazioni degli stati di tensioni di Bonnard, equazioni dei momenti di rotazione efficace del Fellenius, e cosi via.

Là dove poi le schematizzazioni non si presentassero facilmente perseguibili, varranno procedure miste, su sfondo però deduttivo, atte sempre alla riesumazione di " solidi geologici unitari equivalenti ".

Aumentando ancor"a le difficoltà di conversione a "schemi geomatematici ", riaffiora necessariamente la prassi induttiva, l'arbitraria postulazione di "geotipi uniformi " ai quali poter applicare il calcolo per tentativi.

Si perviene sempre a "geoequivalenze unitarie", e dato il loro carattere essenzialmente induttivo, si tratterà più propriamente di "quasi equivalenze $"$.

$\mathrm{E}$ in queste ultime formulazioni "esula», come conseguenza di metodo, il preliminare e fondamentale esame genetico, da cui dipartire 
invece per rintracciare e localizzare l'origine di frana, e le subordinate traiettorie di propagazione.

A questa arbitrarietà d'impostazione ne conseguono purtroppo altre, più o meno implicite, a catena: ove una rottura avvenga, un semplice presupposto isotropico (caratteristiche del suolo uguali in ogni punto e direzione) causa, per ragioni di simmetria, possibilità di rottura propagantesi lungo superfici geometrizzate (ad es. cilindriche), a generatrici parallele ad una certa dimensione, per ivi incanalare lo scorrimento; un tutto che astrattamente torna, tranne che per la realtà.

Tra le più invalse s.d.s. imposte o prefissate, si ricordano quelle di maggiore impiego: Frontard, Rendulich, Patterson, Collin, superficie di scorrimento circolari, logaritmiche, cicloidali, ecc.

L'ipotesi della s.d.s. aspirale (v. Fig. $3 a, 3 b, 3 c, 3 d$ ), offre il vantaggio che tutte le risultanti delle forze normali e di attrito, passano per il punto asintotico $M$, non generando alcun momento di rotazione.

Nell'equazione dei momenti hanno importanza i momenti dovuti solo al peso proprio, carico applicato, coesione.

Sia il momento di rotazione dovuto alla coesione $(K)$ lungo la s.d.s., che gli altri sono ben calcolabili; di qui discende l'equazione di equilibrio.

Se il materiale è privo di coesione si deve esaminare il senso dei movimenti provocati dal peso proprio, dai carichi applicati, dalla pressione di flusso e dalla spinta dell'acqua sotterranea.

Qualora vi sia una superficie la cui direzione di scorrimento coincida col senso rotatorio del momento, "sussisterà il pericolo di frana ".

La spirale, si osservi, si apre nel senso di moto di frana: il raggio della curva di scorrimento cresce dall'alto verso il basso, o, se si vuole, la s.d.s. fa parte di una spirale che va restringendosi verso l'alto.

Con una "s.d.s. circolare ", occorre fissare il centro di essa per diversi angoli di scarpata e diversi coefficienti di coesione, e detto centro si può trovare in più modi.

Nel caso ideale di nessun attrito interno e coesione, la s.d.s. è una "retta" che coincide con la scarpata $(o-\alpha)$.

Definita la s.d.s. critica $M_{1}$ (v. Fig. $4 a, 4 b$ ), si passa a considerare il volume del terreno che preme su di essa, e da qui, come vedremo, si procede per i "puntellamenti elettrici" - rafforzamenti invisibili ma efficaci - impermealizzando dapprima la parte esposta alle variazioni atmosferiche fino ad una certa profondità.

A seconda del tipo litologico, dell'angolo di scarpata, dell'angolo di attrito interno e della coesione, si risale al cerchio $M_{1}$ e relativo raggio: si delimita, a tetto, la striscia di terreno alterabile, e quindi il volume 


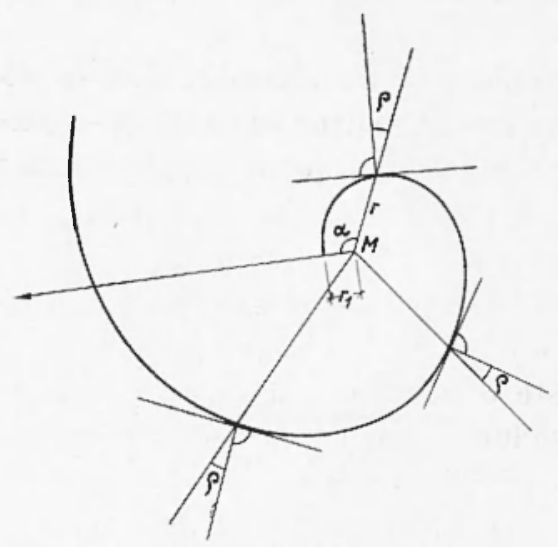

Fig. $3 a$ - Elementi di una spirale lo. garitmica. Equazione della spirale: $r=r_{1} e^{\alpha \operatorname{tg} \rho}$.

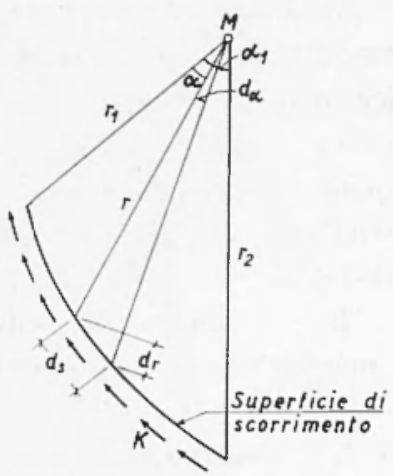

Fir. $3 b$ - siuperfici di scorrimento a forma di spirale logaritmica. Momento di coesionr;

$$
H_{k}=k \frac{1}{2 \operatorname{tg} \varrho}\left(r_{2}^{2}-r_{1}^{2}\right) \text {. }
$$
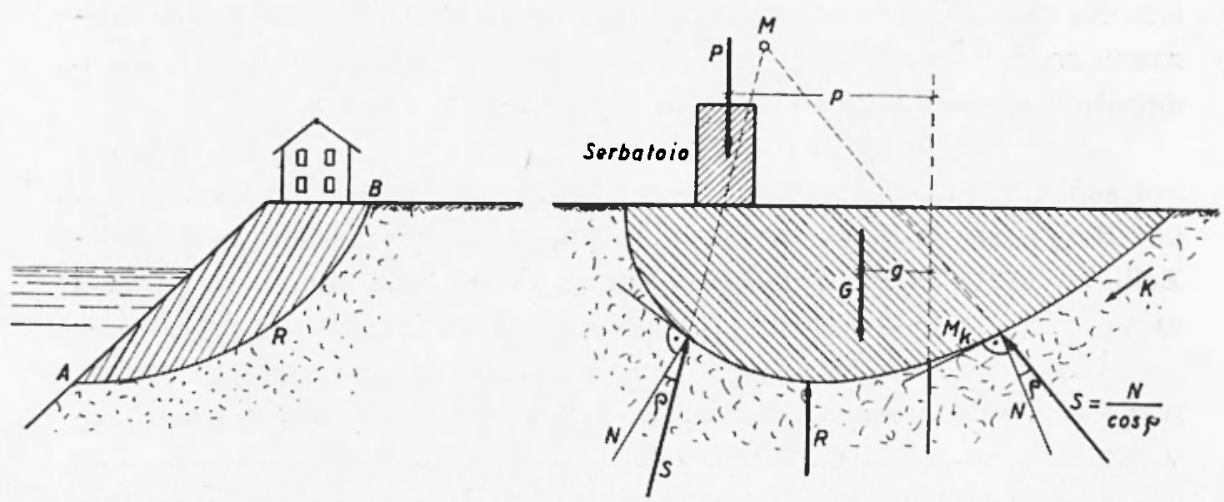

Fig. $3 c$ e $3 l$ - Superfiei di scorrimento a forma di spirale logaritmica. $R$ - Superficie di scorrimento a forma di spirale logaritmica. $-G$ - Peso proprio. $-P=$ Carico applicato.

La "spirale logaritmica " è la famosa curva della "crescenza armoniosa" che si realizza in natura, come nelle conchiglie marine, nella disposizione dei semi del girasole e degli elementi d'una pigna (le cosidette curve della vita del Cook, dell'evoluzione dello spirito di Goethe, o le "curve della morte nel nostro caso). 
cilindrico corrispondente (come verrà detto nella seconda parte "normativa elettrogenetica ").

L'assunzione di forme " ubicazioni arbitrarie di s.d.s. dettate da considerazioni tentativistiche di morfologie tettoniche cinematiche-considerazioni estrinseche alla concretezza del fenomeno -- permetterà i

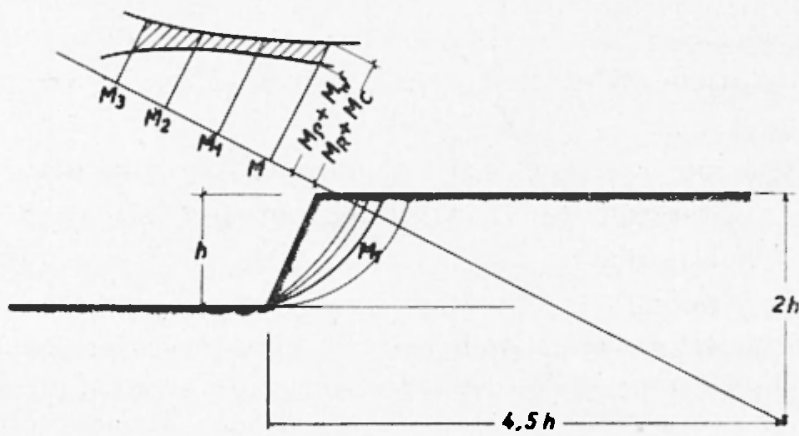

Fig. 4 - Puntellamenti elettrici

computi di "bilanci energetici» (relativi a tali superficie), tra forze attive favorenti il moto e forze resistenti contrastanti, ma essi rispecchieranno valori fittizi di bilanci condizionati, e pertanto porteranno a dubbie conseguenze (v. Fig. 1a e Fig. 4).

4. Le reali s.d.s. critiche (meno resistenti e quindi più pericolose), superficie che poi in genere si realizzano, scaturiscono da origini ben definite da tante azioni di "tettonica dinamica", riesumabili-almeno nei motivi principali - solo da un vistoso corredo di dati di profondità, dati che si possono ottenere in più modi: dai fori di sonda trivellati, non solo limitatamente al prelievo di carote, come d'uso, ma con rilevamenti di pressioni "bottoms" (in fasi di avanzamento e finali, fino alle "failure surfaces"), di pressioni neutre laterali, di tutta una spazialità insomma di sollecitazioni meccaniche in situ.

Dobbiamo accedere pertanto al riconoscinento (come nella telesismica) dell' " ipocentro di frana ", del meccanismo genetico localizzato e maturantesi pre-rottura, e da rilevarsi possibilmente in questo stato anche in quei punti di minima energia potenziale che caratterizzano o denunciano invece le configurazioni stabili di equilibrio maggiormente soggetti a complesse vicende dinamiche (denunciabili già dai minimi spostamenti a carattere premonitorio registrati ad es. con un sismografo-vibrografo). 


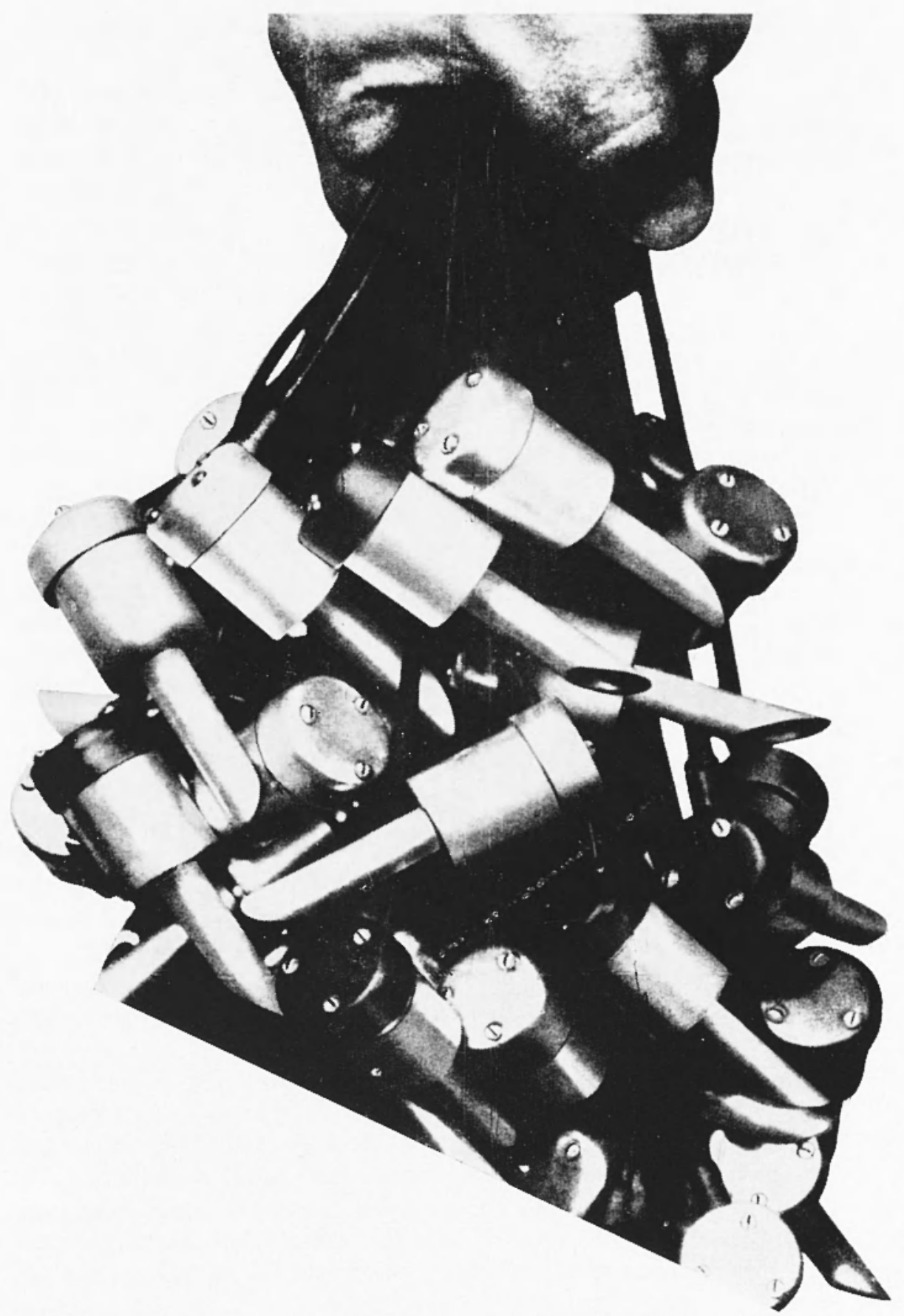

Foto 3 - llall-sears. Inc. 2424 Branard-Houston Texas. Frequenze standard: 14-30 cps. Peso 2 oz. Per tutte le applicazioni su terreno: forte riduzione di personale ed equipaggianento sono rese possibili dal IIS.J. 
Agli aspetti di geoconsistenze labili si dovranno aggiungere quelle salde $\mathrm{ma}$ in stato di recettività di sollecitazioni aggressive.

Premonitoria è la "vibrazionalità » di questi stati particolari, ipocentri di frana, da cui si dipartono le vere s.d.s. lesive; altrimenti non si saprà mai dove fissare l'origine di tali cammini, a meno di raghe ipotesi.

Tali "ipocentri di frana" sono riferibili, si è detto, sia alle consistenze strutturistiche (mono-bi-trifasiche), sia alle geomodellature, sia ad entrambe le cause.

Le sollecitazioni nei complessi "monofasici " possono attivare piani di scorrimento intergranulari (con relativo spargimento di scaglie cristalline lungo questi piani), o "cedimenti intercristallini ".

La bassa pressione o la rapidità applicativa dei carichi favoriscono, secondo Von Karman, il moto intergranulare (genetica geo-molecolare) l'alta pressione o il lento incremento di carico, lo slittamento intercristallino.

La struttura scagliosa ad es. dei minerali argillosi (scaglie isorientate in una direzione preferenziale) a seguito d'una qualsiasi deformazione plastica delle scaglie, comporta lo scorrimento delle une sulle altre, scorrimento favorito dai veli acquiferi aderenti (come convalidano le analisi a raggi Roentgen).

In particolare, la struttura fine di tutti i minerali di argilla presenta ('ostantemente reticoli stratificati caratteristici ad alto potere di scissione.

Cosi mentre nella caolinite $\mathrm{i}$ singoli piani stratificati dei reticoli sono rigidamente interconnessi, nella montmorillonite (componente tipica della bentonite), tali piani sono invece disposti in modo da facilitare la mobilità, possono raddoppiare la loro distanza e l'acqua vicina penetrare negli interstizi.

Dalle basi legate in modo sostituibile nei minerali d'argilla cristallini (qualità e natura chimica), dipende il potere rigonfiante in acqua, elemento d'importanza eccezionale nella geostatica.

La somma delle basi legate $S$ in modo sostituibile, è alta per le bentoniti (alta capacità di scambio che si utilizza nella elettrogeosmosi), e quella monovalente di sodio può assorbire molta acqua, mentre ciò non accade per i calcari e meno ancora per la silice a basso valore $S$.

L'aliquota argillosa costituisce dunque pericoli di frana, almeno fino a quando non interviene "l'elettricità antifrana "!

Negli ipocentri bi o trifasici, la fessurazione può manifestarsi in fase iniziale capillare, con forze e momenti attivi di trascinamento progredienti, che diventano rotatori fino al completo distacco. 
Nei moti intercristallini entrano in gioco addirittura "gli atomi", fino agli slittamenti e conseguenti rotture di genetica atomistica (deformazioni compressive localizzate, eliminazione dei pori, e poi ancora "piani di slittamento "), in sostanza una genetica massiva.

Sedimenti a tessitura rada provocano ipocentri bifasici, che non si attiverebbero con disposizione densa, altra genetica massiva.

Si avvertono perfino fenomeni di dissoluzione selettiva dello scheletro roccioso (effetto Riecke), a seconda delle tensioni agenti (genetica mista).

Cosi dagli ipocentri intrinseci monofasici si passa a quelli estrinseci polifasici, e infine ai geometrici (focolai lineari per rottura di faglia, superficiali per rotture di scorrimento, spaziali per rottura di volumi).

I computi dei bilanci di forze si eseguono secondo le solite norme (una volta rimosse ubicazioni e forme arbitrarie), decomponendo le forze in direzione perpendicolare e orizzontale rispetto alle s.d.s. prescelte, ritenendo efficaci quelle orizzontali, in quanto le forze attive perpendicolari alle s.d.s. vengono equilibrate dalle corrispondenti reazioni del terreno.

Dal rapporto tra la sommatoria delle componenti orizzontali e la sommatoria delle forze attive, si risale, come è noto, al grado di sieurezza $\eta$; se $\eta$ è minore 1 apparirà la "instabilità " del complesso, se $\eta$ ¿ maggiore 1 la "stabilità " dello stesso.

Si noti che il moto che il sistema ipocentrale (centro di massa) può determinare quando è portato ad assumere posizioni prerottura di spostamenti [allontanamenti e ritorni all'origine - moto intermittente, quasi un formicolio di masse vibranti a tendenza vibrografica a basso periodo $\left.\left( \pm 1^{s}\right)-\right]$, può costituire un indice di presagio: "presagio di valanga ".

Oltre ai normali esami microsismici e dromocronici per una più profonda diagnostica e quindi per un più razionale presagio, è opportuno risalire ai rapporti adimensionali più indicativi in questi casi, dal numero di Max che influenza la compressibilità, al numero di Froude che concerne la preponderanza della fase fluida, a quello di Stokes che concerne invece la preponderanza della fase viscosa.

Sorpassandosi ad un certo momenti i limiti elastoplastici, pur in valori contenuti in quelli non ancora distruttivi, i treni di onda incideranno ancor più, specie in ampiezza, con una dissipazione energetica funzione della conduttività elastica della roccia, con assorbimento delle alte frequenze dello spettro energetico dispiegato a seconda il "grado di fragilità » del 
terreno, con influenza di faglie, prerotture, ecc. A questo punto i problemi diventano oltre che difficili, attuali: ci si deve rifare all'equazioni fondamentali dell'onda d'urto (stoss-wellen) dedotte dalle equazioni di conservazione di massa, di quantità di moto ed energia del mezzo. I sistemi generalmente trattati obbediscono ad equazioni differenziali lineari (con l'ipotesi della tensione abbastanza piccola in ogni caso, affinché la forza elastica di richiamo sia proporzionale alla deformazione): ma i problemi della propagazione delle "onde di tensione " (v. Fig. 2) in un mezzo "non lineare", hanno permesso finora soluzioni in casi particolari, conseguite, a partire dai risultati di Riemann-Hugoniot, della scuola russa di Rahmatulin-Sciapiro. I risultati di Kristianovic sulla propagazione delle onde lunghe nei canali e nei fiumi si possono riferire, senza sostanziali modifiche, alle oscillazioni elastiche non lineari nelle sbarre ("onde di scarico" diffuse in mezzi anelastici). Compiti attuali, sulla scia delle più alte impostazioni teoriche sono essenzialmente sperimentali; specifici per poter proseguire poi, oltre tutto, nelle necessarie ricognizioni teoretiche ancora a carattere preliminare. Alludiamo al riconoscimento delle reazioni "quasi-elastiche " dei suoli per effetto di carichi dinamici (specie transitori), da livelli estremamente bassi di tensione a quelli via via maggiori; al riconoscimento delle proprietà visco-elastiche con differenze di contenuto d'umidità di vuoti, ed altro; alludiamo infine ai moti nei mezzi plastici (frequenze basse), alle eterogeneità dei suoli meno appariscenti presentatesi con differenti (notevoli talvolta) proprietà meccaniche, e ad altro ancora che qui omettiamo per brevità.

I vibrograf portatili ("ausculteurs dynamiques ", " accelerografi ") ad installazione permanente di massima nella zona sospetta, come i clinografi e gli estensimetri (jauge de contraintes), permetteranno di risalire (con l'ausilio degli altri dati accumulati) alle cause determinanti, [ubicazione fissa o migrante, consistenze puntiformi, lineari, areali, volumetriche e loro evoluzionalità (v. Foto 2)].

Queste vibrazioni elastiche, oltre a costituire "indici " o "presagi " ipocentrali, fanno corpo del processo stesso franante a seconda i coefficienti energetici in gioco, contributi quindi importanti di dissesto, specie se "risonanti".

Lo stato del mezzo ha in questo (aso caratteristica tensoriale (del $2^{\circ}$ ordine) non essendo più una semplice pressione scalare come nei fluidi.

Un potente ausilio fisico-geo-gnostico appare "l'analisi fotoelasticimetrica ".

In caso di elasticità piana, la ricerca sperimentale delle tensioni interne porta a stabilire, in un punto qualsiusi del test, l'angolo che una delle 
due tensioni principali fa con una direzione fissa (angolo che si ottiene facilmente ponendo la provetta tra due nicols incrociati), e per la legge di Wertheim si ricavano quindi i valori delle tensioni in un punto qualsiasi con tecniche operative molto semplici.

La determinazione delle tensioni interne è uno dei problemi fondamentali come in altri campi dell'Ingegneria.

La teoria generale dell'elasticità conduce, in tali problemi, all'integrazione di un sistema di equazioni a derivate parziali che non si sanno integrare, se non in un numero piccolissimo di casi e con approssimazioni.

Da qui l'importanza di poter risalire sperimentalmente alle "tensioni interne $"$.

Tutto ciò può essere causato dall'esplosione di una polveriera, dalla cristallizzazione di una soluzione soprasatura, dal "break " a percossa dirompente ad asse orientato (risultante dalle sollecitazioni), rilevabile con caratteristiche figure ottico-meccaniche in lastra trasparente orientata normalmente all'asse. Se la risultante è verticale, guardando attraverso nicols incrociati, appare un cono centrale scuro di rivoluzione, ad asse pure verticale, aprentesi verso il basso (dalla parte opposta della percossa), denunziante una "struttura elastica indenne" (il grado di apertura essendo funzione delle costanti di Lamè). Susseguenti aperture coniche chiare (simmetriche, coassiali, concentriche) denunziano parti di «struttura alterata" per le tensioni agenti. Successivamente si ripetono aperture scure - come le prime.

Queste vicende ottiche rispecchiano fedelmente $i$ motivi lesivi concentrici e radiali rispetto al vertice del cono, allorquando pressioni e tensioni oltrepassano i limiti elastici.

Se i mezzi trattati sono plastici, i bulbi di pressione appariranno più appiattiti dei precedenti, potendosi provocare persino, secondo Prandt, ellissoidi di piena rottura locale.

\section{5. $E G E$ (Elettrogetto).}

A questo punto ci fermiamo per illustrare rapidamente i metodi di ristrutturizzazione: alludiamo soprattutto alle applicazioni elettrogeocinetiche, elettrogeosmotiche dapprima (EGO), culminanti poi negli elettrogetti (EGE) fra cui l'elettrogetto integrale (EGEI), e l'EGEI unito con il piezoanodo e il wellpoint-catodo.

Fin dalla sua scoperta l'EGO costituì una semplice curiosità fisica, vennero più tardi le utilizzazioni in problemi di riduzione di acque telluriche (libere, disperse, adsorbite, incorporate nei suoli), fino ai punti di massima densità volumetrica del terreno (punti di Proctor), elettro- 

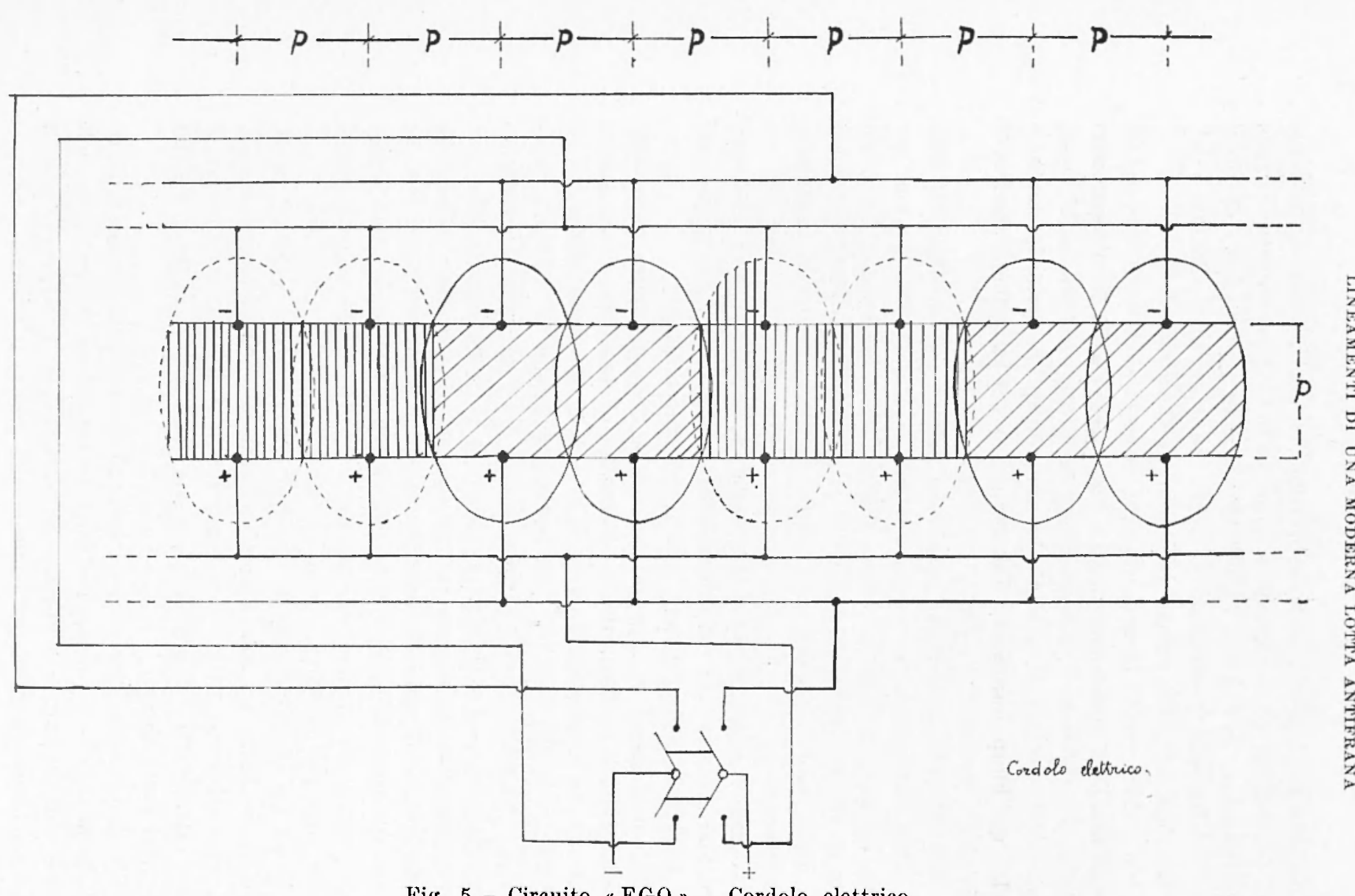

Gli elettrodi metalliei, e i relativi "imbuti di tensione" nel suolo sono da evitare per non subire le conseguenze della "cor. rente pericolosa" (1/20 . 1), se non si è provvisti di gambali e guantoni di gomma. l.a "resistenza ohmica n media di un corpo umano oscilla individualmente, e a seconda lo stato fisiologico. Per la valutazione basta riferirsi alla "resistenza di diffusione nel terreno di un piede umano: $R=2 / 4 \sigma d$ ( $d=$ diametro medio della pianta del piede $\sim 20 \mathrm{~cm}, \sigma-$ conduttività media del terreno $\sim 10^{-4}$ siemens/cm.), quindi $R \leqslant 250 \Omega$, e per ambo i piedi $\sim 500 \Omega-$ Siccome lo ten. sioni sono $\gg 25$ Volts (valore tollerabile con i dati esposti) queste norme di antipericolosità, devono sempre applicarsi. 
prosciugamenti, aggottamenti irreversibili e no, deviazione di flussi idrici (deviazioni planimetriche e altimetriche), abbassamenti di falde, deplastizzazione di limi e di banchi terrosi soffici, "transfert " di sforzi eagenti dell'acqua interstiziale dei pori (pore-pressure) alle fasi solid.

Gli inizi dell'EGO furono però proprio "antifrana »!

Poi l'EGO rivelò la sua capacità impermeabilizzante, indurente, consolidante, in "geoelettrocircuiti " alimentati da c.c. con particolari elettrodi metallici (a depolarizzazione catodica), elettrodi pieni, astiformi, tubolari, forellati, a graticcio forme varie, in serie, in parallelo situate a profondità variabili dalla superficie del suolo fino a diecine di metri (elettrodologia) (v. Fig. 5 "Cordolo elettrico »).

Elettroidromigrazioni nei geocircuiti coinvolgono forti legami adesivi, alterazioni geochimiche, sostituzione di idrogenioni con ioni più stabili, scambi ionici tra soluzioni anodicamente immesse, e complessi terrosi di adsorbimento, superamento di minime permeanze, sbloccaggi vari, idrici, ionici, gassosi, tutti effetti preclusi alle azioni migratorie idromeccaniche.

A causa di neoeffetti relegati in ambiti perielettrodici per insorgenti migrazioni autigene, ivi più intense che altrove, si formano spesso barriere antistanti agli elettrodi, quindi schermi limitativi.

Tali neoformazioni, seppure circoscritte, trovano d'altra parte specifico impiego in problemi di "localizzazione ", "antifrana di distacco", "antifrana di rottura di fondo", "di pendice", "antisfaldamento di scarpata", nei pali "ALU" (pali fasciati di lamine di alluminio anodizzate), "consolidamento " a zone anulari dei "pali sospesi trivellati" concentriche ai pali stessi tali da ampliare il carico ammissibile, con forte permanente incremento di attrito laterale, dispendi di appena qualche $\mathrm{kwh} / \mathrm{m}$ (lineare di mantello metallico sottile).

Questo ristretto campo di azione EGO, nella sua prima forma EGO I, venne poi parzialmente rimosso con la scelta, oltre che di idonei metalli polari, di additivi anodici di soluzioni ioniche (EGO II).

Dopo un certo periodo di ulteriori affinamenti e progressi EGO, si addivenne infine ad una diversa modalita applicativa elettrogeocinetica, "l'elettrogetto EGE" iniezione elettrica di leganti, che non si avvale di interazioni geochimiche, e comporta invece elettroiniettività di leganti precostituiti.

L'elettrogeochimismo cede il posto all'elettrogeoconvogliamento di leganti, processo che si avvale dell'EGO, essendone nel tempo stesso nettamente differenziato e a più ampia possibilità di impiego (v. Foto 4 "preconsolidamento EGE per rimozione di opere fatiscenti"). 
Invero l'EGE compendia e l'EGO e il piezogetto (iniezioni queste nltime a semplice pressione o depressione idraulica o meccanica); si avvale di entrambi fondendoli in una unica cospirante azione, sia con pressione positiva anodica (piezo-anodo), che con pressione negativa catodica (Wellpoint-catodo).

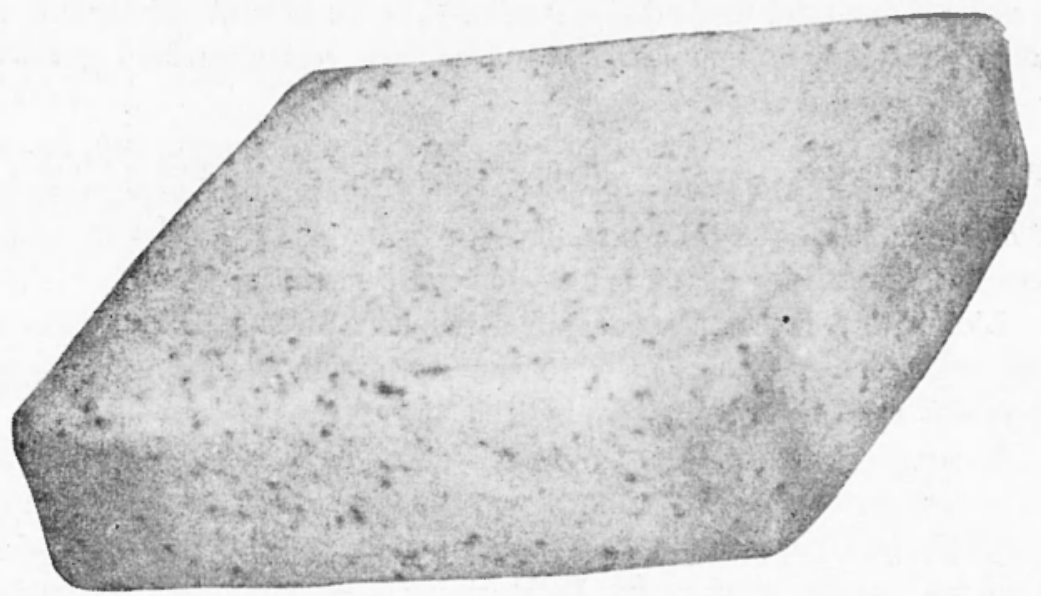

Foto 4 - Arenaria nubica elettrosilicatizzata (Egitto). - Relativi incrementi ponderali, di resistenza meccanica (pietrificazione). I geli dei silicati insolubili, a tenore elevato in acido silicico sono a perfetta otturazione dei pori e crepe del terreno. Si è preferito qui l'uso del silicato di alluminio (rispetto a quello di sodio) unsolubile, a bassa viscosità dei sali (quasi come l'acqua). Solo dopo iniettata la soluzione diluita, (espellendo prima l'acqua contenuta nei pori pretrattamento $\mathrm{EGO),} \mathrm{hopo} \mathrm{un} \mathrm{certo} \mathrm{tempo,} \mathrm{a} \mathrm{causa} \mathrm{della} \mathrm{polimeriz-}$ zazione dei colloidi del silicato, la viscosità incomincia a crescere, e al termine del processo polimerizzante l'intera massa si solidifica in un gelo uniforme (struttura a fascine).

I sistemi piezo-iniettivi standard (di sospensioni, emulsioni, soluzioni chimiche) si possono trasformare in "piezo-elettroiniettivi", ampliando $\mathrm{i}$ "ranges " litologici, reologici, porosimetrici di imbibizione diffusiva o dislocativa dei leganti; in "direzionati squeezings " di iniettato (spazialmente definiti dai noti parallelepipedi geobipolari in funzione delle ampiezze di testata $o$ eteropolari) senza interazioni di geochismimo.

Le normali piezo-iniezioni di cemento anche se a titolo di ipotesi dovessero raggiungere le peculiarità degli "squeezings " EGE, non potrebbero certo modificare la struttura chimico-fisico-minerale del permeato, come si realizza invece con l'EGE. 
Gli scheletri reticolari cementizi post-iniettati non si saldano mai, non monolitizzano con la matrice terrosa (coerente o incoerente che sia in partenza), rimangono parzialmente "sospesi " in essa lasciando indenne parte dei canalicoli, porosità-carie, discontinuità di varia natura.

Né miglioramenti si possono conseguire, nonostante tutte le scelte di cementi iniettivi [colloidali, ultrafini $(\phi=10 \mu)$ cementi-argilla], soluzioni tissotropiche, emulsioni bitumose, pur così insensibili quest'ultime alle acque corrosive.

I "piezo-iniettati cementizi " valgono limitatamente per macrocolmate parziali di fessure, diaclasi, cavità, giunti dislocati, prossimi all'iniettore; né è possibile direzionare o convogliare i getti in ambiti prestabiliti, getti per di più spesso dispersi o deviati a caso.

L'effetto della colmatazione iniettiva normale è parziale e discontinuo nella sua reticolazione matriciale, originando un "neogeostato" a porosità ridotta, trattabile quindi elettrogeocinematicamente.

Il campo di azione EGO-EGE non rimane allora relegato o confinato solo in preesistenti definiti ambiti porosimetrici. Esso è integrativo dei " macropiezici ", per tutti i possibili escursus discontinui, e ciò costituisce un nuovo campo applicativo EGE da noi recentemente instaurato (Brev. sud afr.).

Accenniamo inoltre ad un'altra modalità di intervento, non ancora definitivamente a punto: la "tensiattivazione " delle soluzioni iniettabili, valida purché si riesca ad evitare l'assorbimento selettivo delle fasi solide (bagnabilità preferenziale relativa a quel dato fluido).

Additivi tensioattivi (es. sistepal 4) a debole concentrazione (v. n. di Weber regolante l'influenza della tensione superficiale sul moto fluido), possono attenuare sino al $30 \%$ ed oltre le tensioni interfaciali o interfasali nelle terre umide (il cui ordine di grandezza generico è di una diecina di dine/cm, o anche più).

Si esaltano ancora in tal modo le "forze di scardinamento " delle acque legate al sottosuolo trattato, forze risultanti oltre che da pressioni e depressioni meccaniche, anche da pressioni per convezioni controioniche diffuse per tensioattivazioni (sembrano più efficaci i tensioattivi anionici), incidenti direttamente sugli angoli di contatto fra fasi diverse, la cui entità di azione è legata alla selettività adsorbitiva delle soluzioni, a seconda pure dei componenti di fase solida in giuoco.

Il "pompaggio catodico ritmico" (altra modalità operativa da noi introdotta), si avvale - come abbiamo mostrato di recente - particolarmente del piezo-anodo, oltre che dagli iniettati tensioattivi, del- 
l'additiva aspirazione a vuoto (Well-point), per un optimum di stokage di iniettato.

Da una preliminare rimozione dell'acqua tellurica libera e legata, fino ai films idrici più adesivi in terreni umidi (eduzione oltre che di acque libere pendolari anche dei gusci adsorbitivi più esterni, il che ci preserva di avvicinarsi troppo "all'umidità naturale irriducibile " per rimanere negli intorni igrometrici dei punti di Proctor - densità apaprente dell'aggregato massima), si passa al rimpiazzamento del legante.

6. Con queste nuove tecniche inietto-estrattive è facile constatare come il terreno venga ad essere coinvolto in pieno, "ristrutturato", fin nelle sue discontinuità più nascoste.

\section{SCHEMA INDICATIVO}

\section{E. P. (ADIREZIONALE)}

PERIANODICO

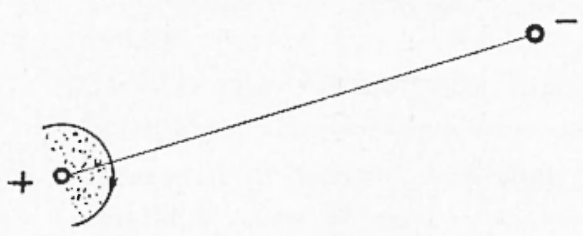

E. I. (DIREZIONATO)

BIPOLARE

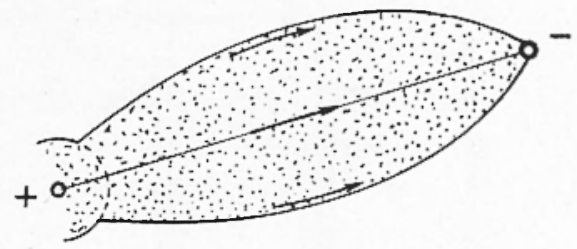

RAPPORTO DI CONSOLIDAMENTO $\frac{\text { E.I. }}{\text { E.P. }}=\sim 15-20$

Fig. 6

Mentre le iniezioni a pressione meccanica - l'EGO ed il Well-point separatamente presi si applicano con ripercussioni circoscritte e limitati raggi d'azione - l'EGE integrale (con la combinazione piezoEGE-Well-point in funzionalità simultanea ed operanti con un unico dispositivo), si libera invece dalle limitazioni specifiche; invade l'intero spazio geo-bipolare in predeterminati orientati contenimenti a massima saturazione di legante, in mezzi qualsiasi, con autoprecipitazioni "in situ s di geli indurenti o monolizzanti con le matrici [v. Fig. 6, Schema EGE parziale (E.P)-EGE integrale (E.I)].

Tra i leganti maggiormente usati e di alto rendimento consolidantisi in situ post-iniezione è da ricordare la soluzione di silicato di sodio con cloruro di calcio determinante nell'elettrosilicatazione(unprocesso 
ormai molto usato e da anni nei Paesi Orientali) l'autoprecipitazione di geli di acido silicico autoindurenti e di alta presa.

Il deus ex machina dell'elettrogeocinetica in genere da tenere presente è l'apparizione funzionale di un vero prodigioso parametro "la permeabilità elettrica $K e$ n, integrativa agli effetti di permeazione di quella idraulica $K$.

Mentre nella normale idrodinamica la fluitazione è retta dalla permeabilità idraulica $K$, secondo la legge di Darcy, nell'EGO la " portata " fluida è proporzionale al prodotto della permeabilità elettrica $K e$ per la tensione applicata $E$ al geocircuito (legge di Helmoltz).

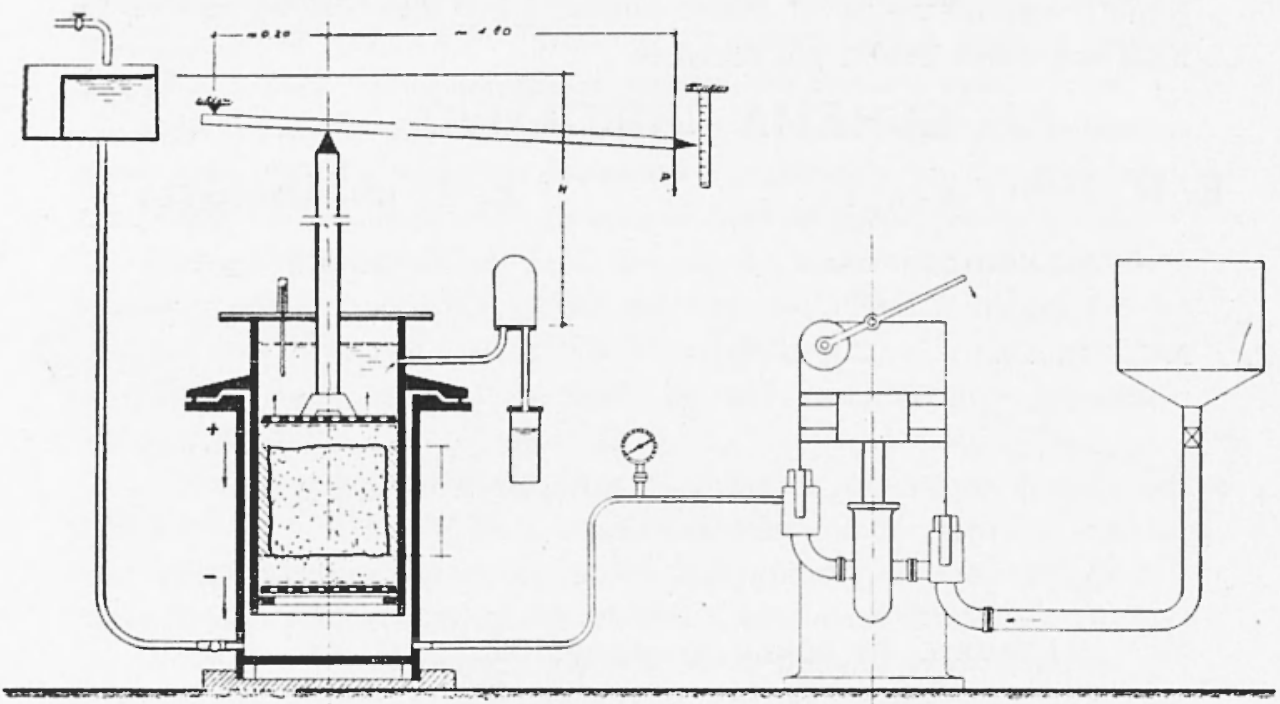

Foto 5 - Iaboratorio Elettrogeotecnica A.B.

Computi comparativi edometrici meccanici e elettrocinetici $K e, K$.

Il flusso idrodinamico è proporzionale al prodotto della permeabilità idraulica $K$ per l'altezza idrostatica (altezza che ha un limite teorico di $10 \mathrm{~m}$ ); nel flusso EGE si ha proporzionalità rispetto al prodotto di $K e$ per la tensione elettrica alimentatrice della c.c. immessa nel terreno ( $E$ limitata dalla termo-elettro-stazionarietà).

Il fattore $K e$ che sovrasta la portata EGE (e pressione relativa) dipendente da $E$ e da altri parametri elettrogeocinetici (doppi strati elettrici in capillari, micropori), sovrasta altresì il fattore $K$ di un rapporto amplificante $\frac{E \cdot K e}{i K}$ e di uno di coazione tra grandezze elettriche e meccaniche da cui viene esaltata l'estraibilità fluido-catodica, 
oltre tutta una ricea serie neofenomenologica di effetti utili (v. Foto 5; Elettroedometro misto).

Una roccia satura poco permeabile, tipo argillite, trattata con un campo elettrico di 1 volt $/ \mathrm{cm}, K=10^{-7} \mathrm{~cm} / \mathrm{sec}, K e=10^{-5} \mathrm{~cm} / \mathrm{sec} /$ Volt/sec educe al catodo cento volte più di quanto non si otterrebbe con gradiente idraulico unitario.

7. Le piezo-iniezioni di soluzioni chimiche vengono praticate in vari modi (si conoscono dieci processi).

Il metodo Langer impiega limpide soluzioni silicatiche alcaline a bassa viscosità, permettenti più facilmente la difísività. La riduzione del $p H$ dosa l'elettrolita e le sue qualità reologiche, sensibilizza i tempi di coalescenza dei geli (regolabili con alta precisione), deidratizza i geli indurenti fino ai cristalli anidri ultramicroscopici.

La determinabilità dei "tempi di precipitazione ", sia dei sali chemioiniettati che degli elettrochemioiniettati (anodizzando il foro immissivo tubolare), è di grande importanza specie nell'EGE per tener conto della " durata iniettiva " saturante (a seconda le porosità del terreno in gioco), e della durata preconsolidante del percorso geobipolare.

La "durata iniettiva " non può naturalmente superare quella precalcolabile di precipitazione del legante in situ. Le post-iniezioni ad intervalli di tempo, non sono del resto sempre consigliabili, se pure qualche volta efficaci.

L'EGE richiede oltretutto per il suo più efficace rendimento (accorgimenti geoelettrici compresi), una serie di ulteriori specifici riconoscimenti petrofisici, sia nel settore della "porosità utile " (tipi intergranulari, vacuolari, fessurativi, consolidati o a sistema indeformabile, non consolidati o a sistema di accatastamento), sia in quello della " permeabilità intrinseca " (la quale dal decimo di milli-Darcy può ascendere a qualche centinaio), che in quello delle "permeabilità relative" (direzionata orizzontale, verticale, anisotropica).

Sono da aggiungere le misurazioni dei "fattori litologici", del "fattore di formazione» e degli "stati di autocementazione» (che si ripercuotono sul reticolo dei canalicoli, dell'ordine del micron questi per le roccie consolidate, fino al mm per le roccie sciolte), le percentuali medie delle saturazioni fluide, le proprietà capillari, superficie specifiche in $\mathrm{m}^{2} / \mathrm{m}^{3}$ (ad es.: con ó diametri di grani di un paio di $\mathrm{mm}$ si ha una certa superficie esposta al fluido in $\mathrm{m}^{2} / \mathrm{m}^{3}$, con gli stessi grani aventi diametri ridotti a qualche centesimo di mm tale superficie specifica si incrementa di diecine di volte). 
A differenza della "piezosilicatazione " dispersiva a diffusione incontrollata e costretta in ambiti ristrettissimi, l'celettropiezosilicatazione " è convogliata a gettata orientata, portata massima, ad alto potere indurente (sia in rocce sciolte, con 50 o più \% di porosità: che in argille o in sabbie meno porose, con circa $35 \%$, che in scisti a marna con $4-10 \%$, fino alle roce cristalline con l' $1 \%$ di pori).

Con "l'EGEI " si piazza legante in quegli ambiti dei mezzi terrosi, là dove si ritiene effettivamente necessario, senza causare deviazioni o perdite imprecisabili di fluidi.

L'estensione e la portata dell'iniettato EGE sono determinate dall'ampiezza interpolare eteronoma, dal carico elettrico immesso (concentrato agli elettrodi), dai carichi elettrici distribuiti lungo " patterns » delle linee di corrente in cui agiscono potenziali $Z$ dei doppi strati elettrici capillari a netta differenza dei soli carichi agenti esterni concentrati che si attuano perciò nei piezogetti antipermeanti, a meno di squeezengs).

"L'EGEI " ̀̀ del tutto insostituibile nei terreni a granulometria naturale fine, o nei terreni riducibili a granulometria post-iniettiva cementizia, là dove la pressione meccanica non ha alcuna efficacia per la ridottissima permeabilità del mezzo, o per la incompleta preliminare macromonolitizzazione cementizia.

Inoltre l'azione della c.c. non si esaurisce in una semplice funzione compressivo-veicolante - come accade con una mera pressione meccanica - al contrario essa intensifica e accelera la gelificazione e la presa, deidratizza fino a trasformare talvolta, diageneticamente, la massa in una vera e propria "pietrificazione ".

Sia con la successiva e contemporanea introduzione nel suolo delle soluzioni silicatiche e elettrolizzanti (bisoli o monosoli), che con l'aspirazione a vuoto al catodo, si possono provocare ulteriori particolari incrementi della "resistenza meccanica del suolo" alla compressione e allo schiacciamento (punte di diecine di atm. nei terreni compressibili), con incrementi della resistenza all'idrodisfacimento per prolungate immersioni.

Per questo potrebbero bastare lievi incrementi di densità silicatica (sia pure senza aggiunta d'elettrolita), viscosità permettendo; ma i ritmi iniettivi, duali agli estrattivi, vengono in genere inevitabilmente rallentati a meno di particolari trattamenti reologici di recente acquisizione.

L'iniettività è ostacolata sia dalle microporosità che dalla viscosità delle soluzioni, pur disponendo di processi atti a fluidificare (water flooding, gas lift), già sperimentati nelle tecnologie petrolifere. 
L'argomento richiederebbe un maggior sviluppo come tanti altri qui toccati, ci basti averne fatto cenno, e suscitato l'interesse (v. Figg. $7 a, 7 b$, schema EGE con modalità piezoanodica e catodica mista).

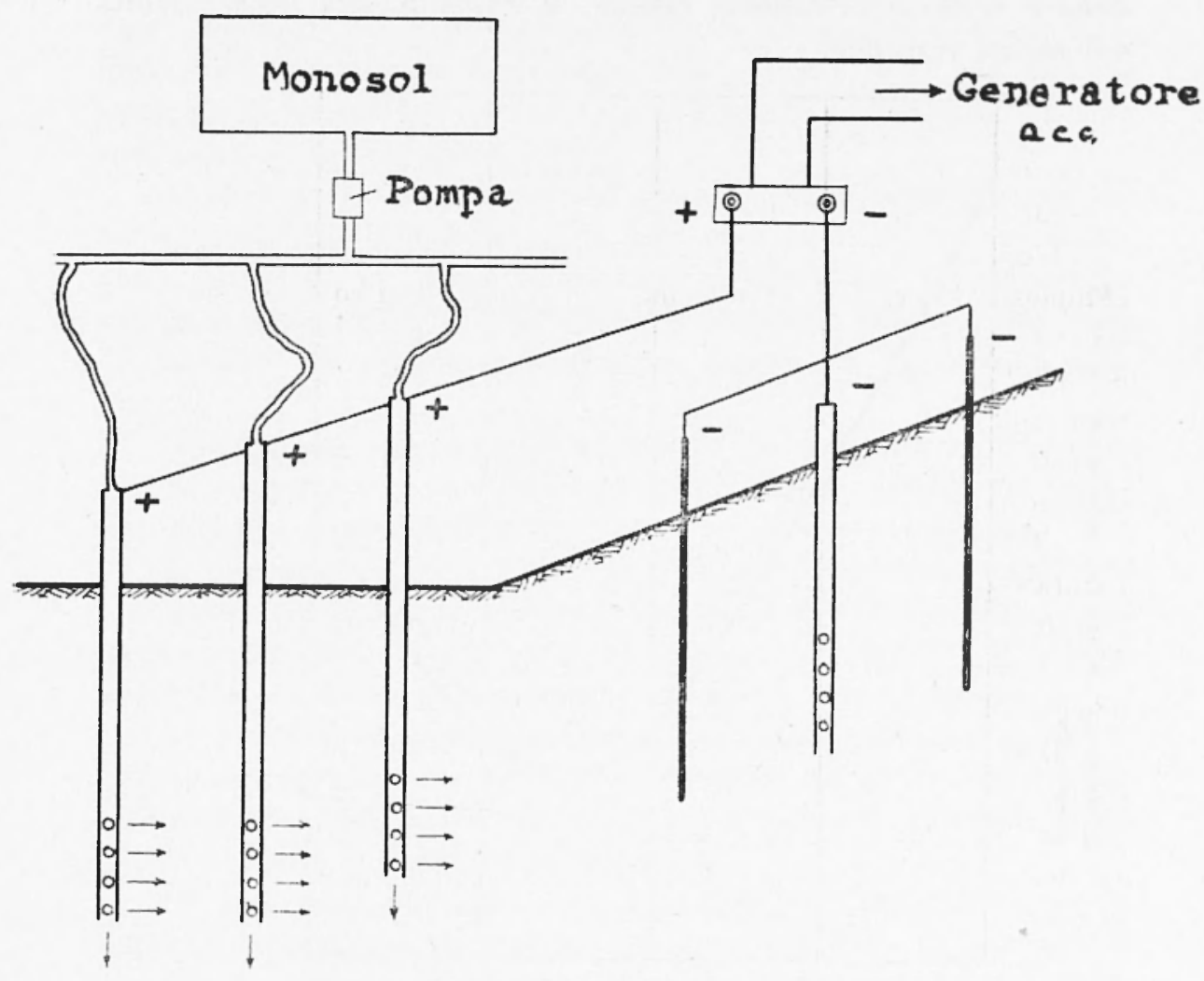

Fig. $7 a-$ EGE (Elettrogetto).

Schema elettrosilicatazione a poinpargio catodico, e catodi in parallelo coa. diuanti (catodi tubolari e pieni). I tubi anodici iniettivi si guarniscono con packer almeno nella parte attivante metallica.

Il " controllo penetrativo del filtrato ", la "saturazione iniettante ", il compimento dello "stato processuale", si fanno in più modi e con verifiche manometriche (un dato grado di pressione residua al catodo), analisi chimiche del fluido estratto, analisi con "traccianti " radioisotopi delle soluzioni aspirate e fuoriuscenti al catodo aspirante.

Questi metodi non distruttivi si utilizzano ora su vasta scala anche per altre misure " in situ "; come le misure del tenore in acqua dei materiali terrosi e della loro densità (assorbimento dei raggi $\mathrm{X}$ proporzionale 
alla densità); misure di umidità basate sulla diffusione dei neutroni (retro diffusione dei neutroni rapidi degli atomi di idrogeno dell'acqua).

L'elettrosilicatazione, avvalendosi di simultanee azioni piezo-anodiche e well-point-catodiche estende la casistica alla quasi totalità dei geotipi trattabili.

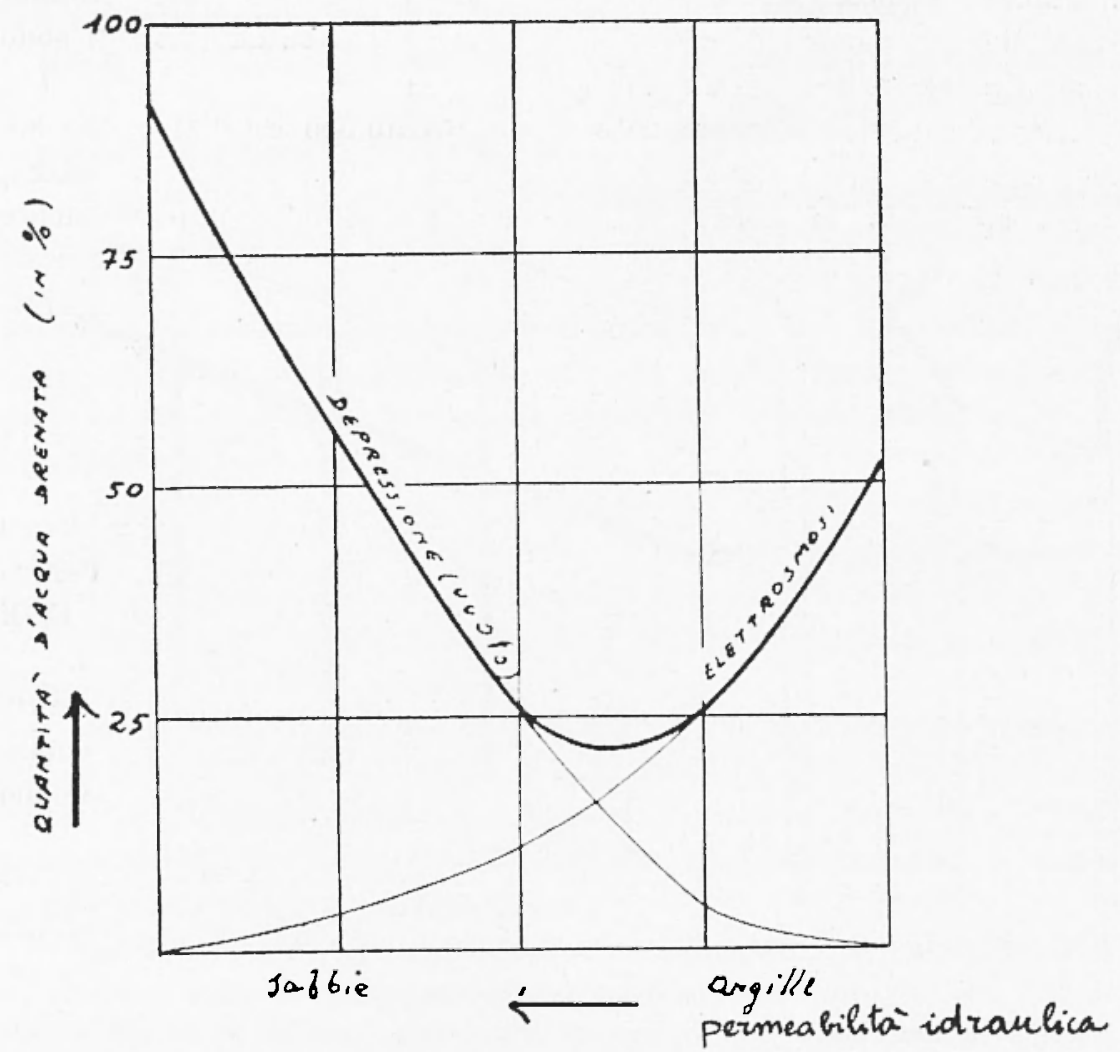

Fig. $7 b$ - Wells-elettro-drenaggi in terreni a litologie miste. Drenaggio completo con EGO-Well point. in terreni sabbio-argillosilinea grossa - Drenaggi parziali con Well point, e EGO separatamente applicati.

I semplici Well-points non possono funzionare nell'argilla, dato che solo nelle sabbie acquifere permettono un rapido abbassamento piezometrico di falda acquifera, con impianti per di più a carattere permanente (pompe di aspirazione centrifughe autoadescanti congiunte ad efficientissime "pompe a vuoto " aspiranti, oppure del tipo centrifugo, ecc.).

Nei "filtri aciculari " le punte filtranti sono infisse mediante acqua in pressione, fino a $10 \mathrm{~atm}$. e poi connesse ad un collettore di aspirazione 
al quale fanno capo motopompe. Tali filtri di aspirazione in sabbie acquifere permettono trattamenti di terreno fino a $K \geqslant 10^{-3} \mathrm{~cm} / \mathrm{sec}$. Abbiamo dimostrato che detti filtri, uniti all'EGE, oltre ad estendere il drenaggio pericatodico, intensificano la circolazione delle soluzioni, migliorano le condizioni di sviluppo delle correnti EGO con diminuzione sia di consumo di corrente che di iniettato (questi risultati sono confermati nella bibliografia russa).

Applicando simultaneamente il vuoto catodico estrattivo con soluzioni dense $\delta_{\mathrm{Sil}}=1,35, \delta_{\mathrm{CaCl}_{2}}=1,25$ (ciò̀ pigra fluidazione EGE), verrà agevolata la stessa immissione anodicofiuida: implicitamente quindi si attua come un "correttivo antiviscoso" (senza l'intervento di tecniche di additivi fluidificanti), aspetto di funzionalità antiviscosa.

Siamo giunti dopo la messa a punto da più di dieci anni del nostro " EGEI " integrale, a un "EGEI piezo-Wellpoint " valevole per tutti i tipi litologici e granulogici.

Il piezogetto, l'EGO, l'EGE, il Well-point, ripetiamo, considerati a sé stanti, rientrano più o meno in limiti che si escludono l'un l'altro, mentre la loro simultaneità porta ad una forma integrativa di "EGE integrale ", l'" $E G E I$ sovra-iniettivo " o "EGEI piezo-Wellpount ".

Le pressioni iniettive e i Well-points, prassi meccaniche, provvedono alla colmatazione dei diametri maggiori dei pori o fessurazioni; mentre l'EGO-EGE prassi elettriche, provvedono alla saturazione dei diametri minori fino alle infime dispersioni.

Gli interventi meccanici non esaurendo mai sufficientemente la impermeabilizzazione, né la riduzione dei coefficienti di permeabilità idraulica (v. iniezioni di cemento), lasciando residui vacuolari nel mezzo trattato, vanno completati con "interventi elettrogeocinetici ", saturando cosi tutte le possibili "discontinuità " naturali (o provocate in più modi, "squeezings " inclusi) del terreno.

Si libera dapprima l'acqua tellurica risospingendola ai catodi iniettati, sostituendola poi con leganti coalescenti in situ, sogeretti a più forze cospiranti di tipo diverso nel consolidamento elettrico.

Si raggiunge, a nostro avviso, la soluzione generale del problema iniettivo.

A fine di garantire la sovrapposizione dei singoli raggi di azione si può procedere, con questo metodo, ad una "iniettività multipla ", ad es. a fasci di "triplofile", colmando piezicamente i pori maggiori (utilizzando le file esterne parallele di ciascuna triplofila), e saturando i pori minori elettrocineticamente (utilizzando la fila mediana). 
Si ha un "EGEI " poli o sovrdiniettivo che sul terreno potrà assumere altre forme, potendo usufruire di uno "stesso dispositivo generatore ", il quale, a sua volta, potrà fungere da semplice iniettore meccanico, da semplice Well-point, da semplice EGO, o, infine, da semplice EGE (le quattro possibili modalità iniettive finora conosciute).

La ricostruzione geostrutturale di terreno passibile di frana, studiato dapprima il suolo nelle sue "equivalenze geomeccaniche" nei suoi ipocentri di frana (s.d.s.), può procedere oggi con bonifiche appropriate integrali di varia natura e di alto rendimento tecnico-economico.

Metodi un po' tutti questi fondamentalmente nuovi, a cominciare dai particolari esami petrofisici e geognostici, dai metodi deduttivi di riesumazione geomeccanica, per arrivare alle terapie meccano-elettrogeotecniche di rafforzamento.

La più razionale geoterapia è quella di rimuovere, mercè tali processi e secondo precise indicazioni operative, le situazioni labili o di insufficienza di georditure e membrature; liberare i volumi rocciosi da stati tensivi eccessivi, regolare le fasi solidofluide nei loro rapporti di compatibilità e di utile ripercussione generale, migliorare le capacità intrinseche di deformazione e di rottura.

Non commentiamo gli interventi standards nelle opere d'arte, rinforzi estrinseci di paramenti soggetti a rapido decadimento e di costo elevato. 